\title{
Screening for cervical Chlamydia trachomatis infections in two Dutch populations
}

\author{
E A P M Thewessen, W I van der Meijden, H J A T Doppenberg, P G H Mulder, \\ J H T Wagenvoort, E Stolz, M F Michel
}

\begin{abstract}
Endocervical cultures for Chlamydia trachomatis and Neisseria gonorrhoeae were taken from 492 women attending an outpatient clinic for sexually transmitted diseases (group I) and 560 women seeking legal abortion (group II). Possible risk factors for $C$ trachomatis infection were evaluated by multivariate analysis. The prevalence rates for $C$ trachomatis and $N$ gonorrhoeae were $7 \cdot 3 \%$ and $2.5 \%$ in group I and $9.4 \%$ and $0.4 \%$ in group II. From multivariate analysis it was found that age ( $p<0.01$ ), number of sexual partners ( $p<$ $0.01)$, abnormal vaginal discharge $(p<0.01)$, and endocervical mucopus $(p=0.02)$ were independently associated with chlamydial infection in group $I$. In the abortion clinic age $(p=0.03)$ and endocervical mucopus $(p=0.03)$ were the only significant independent predictors of $C$ trachomatis. In all women vaginal discharge was collected for Gram staining. A significant higher number of polymorphonuclear cells was seen in the smears of $C$ trachomatis positive women (group I: $p=0.04$; group II: $p=0.03)$. In group II there was also a
\end{abstract}

Department of Clinical Microbiology, University Hospital Rotterdam, Dr. Molewaterplein 40, 3014 GD Rotterdam, The Netherlands

E A P M Thewessen $\dagger$

M F Michel

J H T Wagenvoort

Department of Dermatology and Venereology: University Hospital Rotterdam

W I van der Meijden*

E Stolz

Dr W F Stormclinic, Ebenhaezerstraat 20, 3083 RN Rotterdam, The Netherlands

H J A T Doppenberg

Department of Epidemiology and Biostatistics, Erasmus University Medical School Rotterdam, PO Box 1738, 3000 DR Rotterdam, The Netherlands

P G H Mulder

^Present address: Department of Health, PO Box 3991, Boroko, Papua New Guinea

†Present address: Laboratory Hospitals Gouda, Department of Microbiology, Bleulandweg 3, 2803 HG Gouda, The Netherlands significant association between $C$ trachomatis and Gardnerella type bacterial flora $(p=0.02)$ and the presence of comma-shaped rods $(p=0.04)$. Screening for $C$ trachomatis infection may help to decrease the incidence of (post-abortal) pelvic inflammatory disease. Because screening in abortion clinics is not always possible, decreasing the incidence of postabortal pelvic inflammatory disease could be achieved by using prophylactic antibiotics. Selective use of prophylaxis in high risk patients can minimise costs and the incidence of side effects.

\section{Introduction}

Chlamydia trachomatis is one of the most common sexually transmitted diseases (STDs). Genital infections in women have been associated with cervicitis, endometritis and salpingitis and are therefore a leading cause of infertility and ectopic pregnancy. Women undergoing therapeutic abortion are particularly at risk for infectious complications.

The relationship between clinical signs and symptoms and chlamydial infection is not well established. Chacko and Lovchik $^{1}$ found significantly more chlamydial infections in symptomatic non-pregnant female adolescents than in those without genitourinary symptoms. The presence of genital symptoms was a poor predictor of chlamydial infections in an isolated Alaskan population studied by Toomey $e t$ $a l .{ }^{2}$ Other investigators also found a high prevalence of chlamydial infections among asymptomatic females. . $^{3-5}$

Definitive diagnosis of $C$ trachomatis requires tissue culture isolation. This, however, is costly and not available in all clinical settings. In order to screen selectively it is important to identify risk factors. Young age, a history of more than one sexual partner, a non-barrier method of contraception are reported as important risk factors. ${ }^{1-35-10}$ Also mucopurulent cervicitis, vaginal discharge and hypertrophic cervical ectopy are frequent findings in infected women. ${ }^{1-368-10}$ Non-culture diagnostic tests as cervical Gram stains, vaginal wet smears or PAP smears give inconclusive results as predictive factors for chlamydial infection..$^{34681011}$

The aim of this present study was to determine the 
isolation rate and to evaluate risk factors of cervical chlamydial infection in two defined Dutch populations, that is, women attending an outpatient clinic for sexually transmitted diseases and women visiting an abortion clinic.

\section{Patients and methods}

\section{Subjects}

The study population consisted of 492 women attending the outpatient clinic for sexually transmitted diseases of the University Hospital Rotterdam between July and November 1988 (group I) and 560 women seeking legal abortion at the Dr W F Stormclinic in Rotterdam between June and September 1988 (group II). Women who were taking antibiotics in the two weeks prior to examination were excluded from the study. All women gave informed consent to the work.

\section{Study design}

A detailed history was taken, including age, contraceptive method, number of current partners (in the past 3 months), history of STD and salpingitis, symptoms attributable to lower genital tract infection (abdominal pain and dysuria) and symptoms of urethritis in the sexual partner.

Each patient underwent a complete vaginal and cervical examination. Vaginal discharge, aspect of endocervical secretion, cervical ectopy and abnormalities at bimanual examination were noted. Discharge was labelled as abnormal if it was of excessive volume (present at the introitus) and/or malodorous. Endocervical secretion was either absent, clear or mucopurulent. Ectopy was defined as an area of erythema around the cervical os. Abnormalities at bimanual examination consisted of one of the following findings: uterine or adnexal tenderness or an enlarged adnex.

Endocervical cultures for $C$ trachomatis and Neisseria gonorrhoeae were obtained. Vaginal discharge was collected with a swab and spread onto a glass slide for Gram staining.

\section{Laboratory methods}

Endocervical swabs for chlamydial culture were immediately placed in transport medium. The specimens were stored at $+4^{\circ} \mathrm{C}$ or, when not tested within 24 hours after collection, at $-70^{\circ} \mathrm{C}$. All specimens were processed within 7 days. Chlamydial cultures were performed using cycloheximidetreated HeLa 229 cells as previously described. ${ }^{12}$

Specimens for $N$ gonorrhoeae culture were also taken from the endocervical canal. In group I the swabs were placed into charcoal medium for transport to the laboratory and processed within 4 hours. In the abortion clinic (group II) the swabs were directly streaked onto modified Thayer-Martin medium. The plates were incubated in a $5 \% \mathrm{CO}_{2}$ incubator at $37^{\circ} \mathrm{C}$. Within 24 hours the plates were transported to the laboratory of the University Hospital for further processing. Identification was performed by conventional methods.

All Gram stains were evaluated by the same examiner (WvdM) without knowledge of patient identification or diagnosis. At a magnification of $\times 1,000$ (oil immersion) 10 microscopic fields were examined for polymorphonuclear cells (PMNs), yeasts, comma-shaped rods, and Gardnerella type bacterial flora. When yeasts were seen an extra 10 microscopic fields were searched for pseudohyphae. Candidosis was determined by the presence of yeasts with pseudohyphae. Gardnerella type bacterial flora were present when large numbers of Gram-variable small coccobacilli with a "pepper-and-salt" appearance and clue cells were seen. ${ }^{13}$

No effort was made to look for Trichomonas vaginalis because these are often not visible in a Gram stain.

\section{Statistical analysis}

At first stage, univariate data analysis was performed using the Fisher's exact test, the $\chi^{2}$-test, and the trend test. A p-value less than 0.05 was considered significant.

At second stage, the relationship of multiple subject characteristics with $C$ trachomatis isolation was evaluated by logistic regression analyses. The variables entered were those found to be significant from the univariate analyses of the first stage.

\section{Results}

Six $C$ trachomatis cultures in group II were unreadable owing to disruption of the monolayer. $N$ gonorrhoeae culture results were invalid in three women of group II because of fulminant overgrowth of other microorganisms. Gonorrhoeae cultures were not available for 10 patients in group $I$.

Prevalence rates for $C$ trachomatis and $N$ gonorrhoeae were $7.3 \%(36 / 492)$ and $2.5 \%(12 / 482)$ in

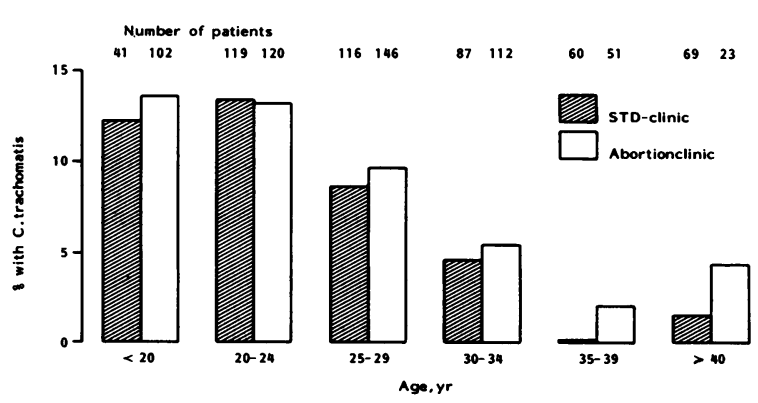

Figure Proportion of patients with cervical $C$ trachomatis infection, by age. 
group I and $9.4 \%(52 / 554)$ and $0.4 \%(2 / 557)$ in group II.

\section{Demographic characteristics}

There was significant association in each of both groups between positive cultures for $C$ trachomatis and age, with younger women having an increased prevalence of infection (fig). In group I, 31 (12.7\%) of those less than 30 years of age were $C$ trachomatis positive and only $5(2.4 \%)$ of those more than 30 years of age $(p<0.01)$. In the abortion clinic the organism was detected in $13.6 \%(44 / 324)$ of women under 30 years of age and in $4.5 \%(8 / 178)$ aged 30 or more $(p<0.01)$.

The method of birth control in patients visiting the STD clinic and the $C$ trachomatis culture results are shown in table 1 . The use of oral contraceptives was associated to a significant extent with isolation of $C$ trachomatis $(p=0.02)$, whereas the use of an IUD or condom was not $(p=0.27$ and $p>0.5)$.

In group I $7 \cdot 3 \%(12 / 164)$ of patients with more than one current sexual partner had a positive $C$ trachomatis culture. Of the 314 women with one or no partner $24(7.6 \%)$ was $C$ trachomatis positive $(p>$ $0.5)$. Amazingly all 45 women with more than one partner in group II had a negative $C$ trachomatis culture, whereas $52(10.4 \%)$ of 499 women with one partner were $C$ trachomatis positive $(p=0.02)$ !

In group I 12 of 111 women with a history of gonorrhoea were $C$ trachomatis culture positive. In

Table 1 Relationship of $C$ trachomatis culture results to method of birth control among women visiting the STD clinic

\begin{tabular}{lcc}
\hline & \multicolumn{2}{l}{ Culture for C trachomatis } \\
\cline { 2 - 3 } Contraceptive practice & Positive & Negative \\
\hline n & 36 & 456 \\
OC & $24(66 \cdot 6)^{\star}$ & $192(42 \cdot 1)$ \\
IUD & $3(8 \cdot 3)$ & $14(3 \cdot 1)$ \\
condom & $3(8 \cdot 3)$ & $38(8 \cdot 3)$ \\
other & $1(2 \cdot 8)$ & $75(16 \cdot 5)$ \\
none & $4(11 \cdot 1)$ & $99(21 \cdot 7)$ \\
unknown & $1(2 \cdot 8)$ & $28(6 \cdot 1)$ \\
pregnant & 0 & $10(2 \cdot 2)$ \\
\hline
\end{tabular}

$\mathrm{OC}=$ oral contraceptives, IUD $=$ intrauterine device.

^Percentage.

Table 2 Isolation of $C$ trachomatis (CT) and $N$ gonorrhoeae (GO) related to symptoms of lower genital tract infection

\begin{tabular}{lllll}
\hline & \multicolumn{4}{l}{ Culture results } \\
\cline { 2 - 5 } Symptoms & \multicolumn{1}{l}{$C T+/ G O+C T+/ G O-C T-/ G O+C T-/ G O-$} \\
\hline $\begin{array}{c}\text { Group I } \\
\text { yes }\end{array}$ & 2 & 11 & 1 & 143 \\
no & 1 & 22 & 7 & 291 \\
Group II & & & 0 & 192 \\
yes & 0 & 24 & 0 & 302 \\
no & 2 & 26 & 0 & \\
\hline
\end{tabular}

group II 10 women reported a history of gonorrhoea. None of them were $C$ trachomatis culture positive. A history of $C$ trachomatis, syphilis, herpes simplex or genital warts was reported by 74 women in group I and nine women in group II. Five of these women in group I and one woman in group II had a positive $C$ trachomatis culture. Fifty patients in group I and 28 patients in group II had a history of salpingitis. Three out of 50 and two out of 28 were $C$ trachomatis culture positive. Neither a history of STD nor salpingitis increased the risk of acquiring chlamydial infection ( $p>0.5$ in both groups).

\section{Symptoms and signs}

Among those negative for $\mathrm{Ngonorrhoeae} \mathrm{there} \mathrm{was} \mathrm{no}$ significant relation between $C$ trachomatis and symptoms of lower genital tract infections in each of both groups (group I: $\mathrm{p}>0.5$; group II: $\mathrm{p}=0.27$ ) (table 2).

Eleven $(21.2 \%)$ of 52 women in group I having a sexual partner with symptoms of urethritis were $C$ trachomatis culture positive, compared with $5.4 \%$ (16/296) of women with a partner without complaints $(\mathrm{p}<0.01)$. Only six women visiting the abortion clinic reported symptoms in the sexual partner. All six were $C$ trachomatis and $N$ gonorrhoeae negative.

Abnormal discharge was noted in 15 of 31 women in group I whose cultures were positive for $C$ trachomatis and negative for $N$ gonorrhoeae. Of the 396 women with both cultures negative abnormal discharge was seen in 93 which difference was statistically significant $(p>0.01)$. In group II this significant difference was not seen.

Excluding all women with gonorrhoea $C$ trachomatis was isolated from $25(12 \cdot 1 \%)$ of 206 women in group I with endocervical exudate characterised as mucopurulent, compared with $6(2.5 \%)$ of 238 with clear or absent secretions $(p<0.01)$. In group II $12.6 \%(36 / 286)$ of women with endocervical mucopus were infected with $C$ trachomatis compared with $5.6 \%(14 / 249)$ of women with normal secretions $(\mathrm{p}<0.01)$.

The presence of cervical ectopy was significantly associated with chlamydial but not with gonococcal infection (table 3). Sixteen percent of the gonorrhoea

Table 3 Isolation of $C$ trachomatis (CT) and N gonorrhoeae (GO) related to cervical ectopy

\begin{tabular}{cllll}
\hline & \multicolumn{4}{l}{ Culture results } \\
\cline { 2 - 5 } Ectopy & \multicolumn{4}{l}{$C T+/ G O+C T+/ G O-C T-/ G O+C T-/ G O-$} \\
\hline Group I & & 20 & 4 & 105 \\
present & 1 & 11 & 5 & 313 \\
absent & 2 & 26 & 0 & 176 \\
Group II & 1 & 23 & 0 & 311 \\
present & 1 & 1 & & \\
absent & 1 &
\end{tabular}


negative women with ectopy in group I were infected with $C$ trachomatis, compared with $3.4 \%$ of women without ectopy $(p<0.01)$. In the abortion clinic $12.9 \%$ of the gonorrhoea negative women with ectopy were infected with $C$ trachomatis, compared with $6.9 \%$ of those without ectopy $(p=0.03)$.

Abnormalities at bimanual examination were not associated with $C$ trachomatis infection $(p>0.5$ in both groups).

\section{Multivariate analysis}

Based on univariate statistical significance seven variables were selected for multivariate logistic regression analysis. Age was entered as a continuous variable. Oral contraceptive use (only in group I), number of current sexual partners (none or one vs two or more), symptoms in the partner, abnormal vaginal discharge, endocervical mucopus, and cervical ectopy were entered as dichotomous variables. All of these variables had valid observations for $388(78.9 \%)$ of the 492 women in group I and for 439 $(78.4 \%)$ of the 560 women in group II. Twenty-eight $(7 \cdot 2 \%)$ respectively $44(10 \%)$ of these were infected with $C$ trachomatis.

Because all 45 women with more than one partner and all six women reporting symptoms in the sexual partner in group II were $C$ trachomatis negative, these variables could not be included in the logistic regression analysis in group II.

In the STD clinic age was independently and negatively associated with chlamydial infection. Number of sexual partners, abnormal vaginal discharge and endocervical mucopus were found to be independently and positively associated. In the abortion clinic age and endocervical mucopus were the only significant predictors of $C$ trachomatis (table 4 ).

\section{Gram stain}

A significant higher number of PMNs was seen in the vaginal smear of $C$ trachomatis positive women than in women with a negative chlamydial culture (group I: $p=0.04$; group II: $p=0.03$ ).

In group II women with Gardnerella type bacterial flora were more frequently infected with $C$ trachomatis $(25 / 178)$ than women with normal secretions $(24 / 332)(p=0.02)$. In group I this difference was not statistically significant $(9 \cdot 7 \%$ versus $5 \cdot 8 \%)$ $(p=0 \cdot 23)$.

Candidosis was found in 25 women in group I and in 27 women of group II. It was not associated with $C$ trachomatis infection ( $p>0.5$ in both groups).

In group II there was a significant association between comma-shaped rods and $C$ trachomatis $(p=0.04$; group I: $p>0.5)$.

\section{Discussion}

The prevalence of $C$ trachomatis and $N$ gonorrhoeae infections varies greatly dependent on the population studied. The differing incidences of cervical infections in STD clinics are dependent on the patient selection criteria used. In 240 partners of men with non-gonococcal urethritis $C$ trachomatis was isolated in $38 \%$ by Paavonen and Vesterinen. ${ }^{3}$ Arya et al ${ }^{14}$ reported a prevalence rate of $33.3 \%$ of $C$ trachomatis and $31.4 \%$ of $N$ gonorrhoeae in 474 unselected women visiting a STD clinic in England. Our prevalence rates in the abortion clinic are comparable to those found by others. Avonts and Piot ${ }^{7}$ detected $C$ trachomatis in $12 \%$ of 125 women and $N$ gonorrhocae in $0.6 \%$ of 161 women seeking abortion in Antwerp, Belgium. Levallois $e t a l^{5}$ reported a rate of $11.4 \%$ of $C$ trachomatis and $0.9 \%$ of $N$ gonorrhoeae among 920 females attending a Canadian Family Planning Clinic for curettage abortion. Whether pregnancy itself is a risk factor for chlamydial infection is unclear, although Bagshaw and Edwards ${ }^{15}$ found such a correlation.

This study confirms that younger women are more likely than older women to have $C$ trachomatis infections. After adjustments for potentially confounding variables, like the use of oral contraceptives, number of current sexual partners or cervical ectopy this association persisted. Chronic $C$ trachomatis infection or constant reinfection over a period of years could account for a protective immune state in older women. Changes in pattern of transmission could also explain this finding.

Table 4 Variables shown by multivariate analysis to be independently associated with chlamydial infection

\begin{tabular}{|c|c|c|c|c|}
\hline Variable & $\begin{array}{l}\text { Regression } \\
\text { coefficient }\end{array}$ & Standard error & pvalue & $\begin{array}{l}\text { Odds ratio } \\
(95 \% C I)\end{array}$ \\
\hline $\begin{array}{l}\text { Group I } \\
\text { constant } \\
\text { age } \\
\text { number of partners } \\
\text { abnormal discharge } \\
\text { endocervical mucopus } \\
\text { group II: } \\
\text { constant } \\
\text { age } \\
\text { endocervical mucopus }\end{array}$ & $\begin{array}{r}-0.487 \\
-0.134 \\
1.602 \\
1.141 \\
1.138 \\
-1.227 \\
-0.055 \\
0.752\end{array}$ & $\begin{array}{l}0.040 \\
0.489 \\
0.432 \\
0.497\end{array}$ & $\begin{array}{r}<0.001 \\
0.001 \\
0.008 \\
0.022\end{array}$ & $\begin{array}{l}0.615 \\
0.875(0.81-0.95) \\
4.963(1.90-12.94) \\
3.131(1.34-7.30) \\
3.120(1.18-8.26) \\
0.293 \\
0.946(0.90-0.99) \\
2.121(1.09-4.14)\end{array}$ \\
\hline
\end{tabular}


We found an increased prevalence of chlamydial infection in women using oral contraceptives as has been documented by others. ${ }^{3}$ This, however, was not an independent risk factor after using loglinear analysis, agreeing with the reports of Bump et al. ${ }^{4}$ One would expect that use of a condom would give some protection from infection. Inconsistent use of a condom could explain our results.

The finding of a positive, independent relation between more than one sexual partner in the previous 3 months and chlamydial infection in the STD clinic is in agreement with the findings of Chacko and Lovchik. ${ }^{1}$ The fact that in the abortion clinic only women with one current partner were infected, could not be explained.

A past history of STD or salpingitis did not significantly increase the risk of acquiring $C$ trachomatis infection, a finding in agreement with earlier studies. ${ }^{15}$ Jones $e t ~ a l^{9}$ even found significantly more chlamydial infection in women without a history of STD, reflecting the theory of protective immunity from prior infection.

Symptoms of lower genital tract infection were not found to be related to chlamydial infection, as has been reported by many authors. ${ }^{2710}$ This finding again stresses the importance of screening women with and without genitourinary symptoms.

Holmes et al ${ }^{16}$ isolated $C$ trachomatis in $68 \%$ of contacts of male patients with nongonococcal urethritis. Consistent with this observation is our finding that significantly more female partners of men with symptoms of urethritis are infected with $C$ trachomatis. This, however, was not found to be an independent risk factor, determined by loglinear analysis.

Abnormal vaginal discharge is one of the major reasons for visiting a STD clinic and in our study we found abnormal discharge in $25.3 \%$ of women in group I. We noted an independent, significant association between cervical chlamydial infection and abnormal vaginal discharge in group $I$ in agreement with Bump et al. ${ }^{4}$ Although abnormal discharge was found in $17.2 \%$ of women visiting the abortion clinic, we did not find such a significant correlation in this group.

We found an independent correlation between endocervical mucopus and the presence of $C$ trachomatis in both populations studied. This confirms the need to treat all women with the clinical sign of mucopurulent endocervical discharge as recommended by the Centers for Disease Control, Division of Sexually Transmitted Diseases. ${ }^{17}$

In agreement with others who used multivariate analyse ${ }^{1018}$ we did not find cervical ectopy to be an independent risk factor for chlamydial infection.

Larsson et $a^{19}$ examined Gram stains for the presence of clue cells and found no correlation with the identification of $C$ trachomatis. Because we did not look for other criteria for bacterial vaginosis (BV) like $\mathrm{pH}>4.5$, positive amine test and clue cells in a wet mount we can not demonstrate a correlation between $\mathrm{BV}$ and $C$ trachomatis. Christiano et al, ${ }^{20}$ however, found such an association.

Our results confirm that $C$ trachomatis is a common STD in the populations studied. Confirmation and treatment of chlamydial infected women may help to decrease the incidence of urethritis in male partners and pelvic inflammatory disease (PID). However, women attending an abortion clinic mostly visit the clinic only once. Decreasing the incidence of postabortal PID in this population could be achieved by using prophylactic antibiotics. To minimise costs and the incidence of side effects selective use of prophylaxis in high-risk patients could be a reasonable alternative. Careful prospective studies, however, are necessary to investigate further the need for routine antibiotic prophylaxis in operations for legal abortions.

The authors are indebted to the physicians and nursing staff of the outpatient clinic for sexually transmitted diseases and the Dr W F Stormclinic for obtaining patient data and specimens. We thank Anne-Marie Meerhoff-Verel, Anita Overkleeft-van de Ree, and Tineke van Rijsoort-Vos for assistance in performing chlamydial and gonococcal cultures. We also thank Margreet Boeren-den Boer and Camille van de Zand for assistance in data assimilation and preparation of the manuscript.

Address for correspondence: Dr E A P M Thewessen, Laboratory Hospitals Gouda, Dept of Microbiology, Bleulandweg 3, 2803 HG Gouda, The Netherlands.

1 Chacko MR, Lovchik JC. Chlamydia trachomatis infection in sexually active adolescents: prevalence and risk factors. Pediatrics 1984;73:836-40.

2 Toomey KE, Rafferty MP, Stamm WE. Unrecognized high prevalence of Chlamydia trachomatis cervical infection in an isolated Alaskan Eskimo population. JAMA 1987;258:53-6.

3 Paavonen J, Vesterinen E. Chlamydia trachomatis in cervicitis and urethritis in women. Scand J Inf Dis 1982;32S:45-54.

4 Bump RC, Sachs LA, Buesching III WJ. Sexually transmissible infectious agents in sexually active and virginal asymptomatic adolescent girls. Pediatrics 1986;77:488-94.

5 Levallois P, Rioux JE, Côté L. Chlamydial infection among females attending an abortion clinic: prevalence and risk factors. Can Med Assoc J 1987;137:33-7.

6 Wood PL, Hobson D, Rees E. Genital infections with Chlamydial trachomatis in women attending an antenatal clinic. Br J Obstet Gynaecol 1984;91:171-6.

7 Avonts $D$, Piot $P$. Genital infections in women undergoing therapeutic abortion. Eur J Obstet Gynec Reprod Biol 1985; 20:53-9.

8 Martin DH, Pastorek JG, Faro S. Risk factors for Chlamydia trachomatis infection in a high risk population of pregnant women. In: Oriel D, Ridgeway G, Schachter J, TaylorRobinson D, Ward M (eds.) Chlamydial infections. Cambridge, Cambridge University Press, 1986;189-92.

9 Jones RB, Mannel JB, Shepard MK, Fisher RR. Recovery of Chlamydia trachomatis from the endometrium of women at 
risk for chlamydial infection. Am J Obstet Gynecol 1986; 155:35-9.

10 Moscicki B, Shafer MA, Millstein SG, Irwin CE Jr., Schachter $\mathrm{J}$. The use and limitations of endocervical Gram stains and mucopurulent cervicitis as predictors for Chlamydia trachomatis in female adolescents. Am J Obstet Gynecol 1987; 157:65-71.

11 Thejls H, Rahm VA, Rosen G, Gnarpe H. Correlation between chlamydia infection and clinical evaluation, vaginal wet smear, and cervical swab test in female adolescents. Am J Obstet Gynecol 1987;157:974-6.

12 Thewessen EAPM, Freundt I, van Rijsoort-Vos JH, Stolz E, Michel MF, Wagenvoort JHT. Comparison of HeLa 229 and McCoy cell culture for detection of Chlamydia trachomatis in clinical specimens. J Clin Microbiol 1989;27:1399-400.

13 Balsdon MJ, Pead L, Taylor GE, Maskell R. Corynebacterium vaginale and vaginitis a controlled trial of treatment. Lancet 1980;i:501-4.

14 Arya OP, Mallinson H, Goddard AD. Epidemiological and clinical correlates of chlamydial infection of the cervix. $\mathrm{Br} J$ Venereal Dis 1981;57:118-24.

15 Bagshaw SN, Edwards D. Risk factors for Chlamydia trachomatis infection of the cervix: a prospective study of 2000 patients at a family planning clinic. $N Z M e d J 1987 ; 100: 401-3$.

16 Holmes KK, Handsfield HH, Wang SP, Wentworth BB, Turck $M$, Anderson JB, Alexander ER. Etiology of nongonococcal urethritis. N Eng J Med 1975;292:1199-205.

17 Division of Sexually Transmitted Diseases, Centre for Prevention Servics, Centers for Disease Control. Chlamydia trachomatis infections: policy guidelines for prevention and control. MMWR 1985;34:30-42.

18 Handsfield HH, Jasman LL, Roberts PL, Hanson VW, Kothenbeutel RL, Stamm WE. Criteria for selective screening for Chlamydia trachomatis infection in women attending family planning clinics. JAMA 1986;255:1730-4.

19 Larsson PG, Bergman B, Forsum U, Platz-Christensen JJ, Pahlson C. Mobiluncus and clue cells as predictors of PID after first-trimester abortion. Acta Obstet Gynecol Scand 1989;68:217-20.

20 Christiano L, Coffetti N, Dalvai G, Lorusso L, Lorenzi M. Bacterial vaginosis: prevalence in outpatients, association with some micro-organisms and laboratory indices. Genitourin Med 1989;65:382-7.

Accepted for publication 10 July 1990 